\title{
Novel rearrangement in the reaction of 5-methyl-5 $H$-iso-indolo [2,1-a]benzimidazole with maleimide derivatives. Stereochemical and X-ray structural study
}

\author{
Zoia Voitenko, ${ }^{\mathrm{a}}$ Volodymyr Lyaskovskyy, ${ }^{\mathrm{a}}$ Jean Gérard Wolf, ${ }^{* \mathrm{~b}}$ and Joël Jaud ${ }^{\mathrm{c}}$ \\ a Department of Chemistry, Kyiv National Taras Shevchenko University, Volodymyrska st. 64, \\ Kyiv, 01033, Ukraine \\ ${ }^{b}$ Synthèse et Physicochimie de Molécules d'Intérêt Biologique, UMR 5068, Université Paul \\ Sabatier, 118 route de Narbonne, F-31062 Toulouse cedex 9, France \\ ${ }^{c}$ CEMES LOE, 29 rue Jeanne Marvig, BP 4347, F-31055 Toulouse cedex 4, France \\ E-mail: wolf@,chimie.ups-tlse.fr
}

\begin{abstract}
In the reaction of 5-methyl-5H-isoindolo[2,1-a]benzimidazole 2 with $N$-arylmaleimides $\mathbf{3 a - f}$ a novel unexpected rearrangement led to the 3- $(E)-1-(2,5$-dioxo-1- $R$-tetrahydro-1H-3-pyrrolyl)-1[2-(1-methyl-1H-benzo[ $d]$ imidazol-2-yl)phenyl]methylidene-1- $R$-2,5-pyrrolidinediones 6a-f. A three-step mechanism including Michael addition, Diels-Alder reaction and skeletal rearrangement of tricyclic 7-azanorbornene derivatives $\mathbf{5 a}-\mathbf{f}$ is proposed to explain the formation of the final products. An X-ray structure analysis of compound $6 \mathbf{6}$ shows the presence of a twisted double bond with a torsion angle of $6.42^{\circ}$. In the ${ }^{1} \mathrm{H}$ NMR spectra the presence of a chirality center and axis in the adducts $\mathbf{6 a}-\mathbf{f}$ implies the presence of atropodiastereomers.
\end{abstract}

Keywords: Atropisomerism, Diels-Alder reaction, cycloaddition, isoindolo[2,1-a]benzimidazole, Michael addition

\section{Introduction}

The study of the chemical properties of isoindole derivatives is an important area in heterocyclic chemistry. ${ }^{1}$ Their most typical reaction is the [4+2]cycloaddition (Diels-Alder reaction), which is well studied for simple isoindoles. ${ }^{1 b, c, 2}$ In contrast, the reactions of the corresponding annulated derivatives with dienophiles are more complex and include not only Michael or DielsAlder reactions but are also usually accompanied with original skeleton rearrangements. ${ }^{3}$

Among the other annulated isoindoles which are much more delocalized, the heterocyclic system of isoindolo[2,1-a]benzimidazole 2 occupies a special place. ${ }^{4}$ This is due to a weak interaction between the isoindolic and the benzimidazolic parts of the molecule. It was thus 
interesting to study their reactions with dienophiles. Herein, we present the original reactions of the 5-methyl-5H-isoindolo[2,1-a]benzimidazole. ${ }^{5}$

\section{Results and Discussion}

The starting isoindole $\mathbf{2}$ was obtained in situ from the 5-methyl-5 $\mathrm{H}$-isoindolo[2,1a]benzimidazolium iodide 1 using triethylamine as a base (Scheme 1). Reaction of 2 with two equivalents of $p$-tolylmaleimide leads to the compound $\mathbf{6 a}$ (71\% yield). Its structure was proved by an X-Ray structure analysis (Figure 1). The analogous compounds (6b-f) were isolated in high yields $(72-85 \%)$ in the reaction with other $N$-arylmaleimides, and completely identified by NMR-, mass-, IR- spectra and elemental analysis.<smiles></smiles>

1

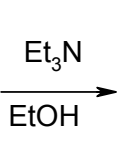<smiles></smiles>

2
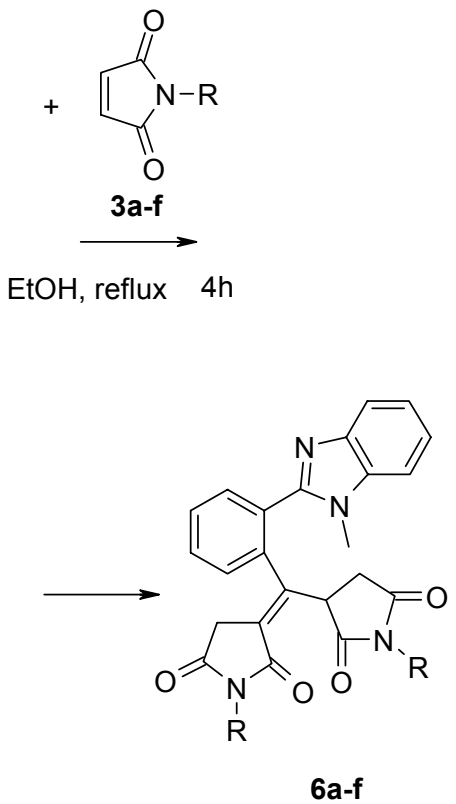

3-6: a R=p- $\mathrm{C}_{6} \mathrm{H}_{4}-\mathrm{Me}, \boldsymbol{b} \mathrm{R}=\mathrm{p}-\mathrm{C}_{6} \mathrm{H}_{4}-\mathrm{OMe}, \mathbf{c} \mathrm{R}=\mathrm{p}-\mathrm{C}_{6} \mathrm{H}_{4}-\mathrm{Br}$, d $\mathrm{R}=\mathrm{p}-\mathrm{C}_{6} \mathrm{H}_{4}-\mathrm{Cl}$, e $\mathrm{R}=\mathrm{m}-\mathrm{C}_{6} \mathrm{H}_{4}-\mathrm{NO}_{2}, \mathbf{f} \mathrm{R}=\mathrm{Ph}$

\section{Scheme 1}

The main feature concerning the $\mathrm{X}$ - ray result is that it permits to ascertain the proposed structural determination. Compound 6a crystallizes with one molecule of chloroform and we observe no noticeable intra or intermolecular short distances. The 5-membered ring $\mathrm{C}(2)-\mathrm{C}(3)-$ $\mathrm{C}(4)-\mathrm{N}(1)-\mathrm{C}(5)$ in the crystal is nearly planar (no atom deflection from the root-mean-square plane $>0.02 \AA)$ whereas the other succinimide ring $\mathrm{C}(13)-\mathrm{C}(14)-\mathrm{C}(15)-\mathrm{N}(2)-\mathrm{C}(16)$ present a slight envelope form with $\mathrm{C}(13)$ situated at $0.134 \AA$ over the root-mean-square plane of the four 
other atoms (no deflection $>0.02 \AA$ ). The tolyl substituents are staggered to the five membered rings (torsion angles are $50.66^{0}$ for $\mathrm{C}(4)-\mathrm{N}(1)-\mathrm{C}(6)-\mathrm{C}(11)$ and $-70.42^{0}$ for $\mathrm{C}(16)-\mathrm{N}(2)-\mathrm{C}(17)-$ $\mathrm{C}(22)$ ) and quite parallel together. The double bond $\mathrm{C}(1)-\mathrm{C}(2)$ is twisted (torsion angles $\mathrm{C}(24)-$ $\mathrm{C}(1)-\mathrm{C}(2)-\mathrm{C}(3)$ is $\left.6.21^{\circ}\right)$ and is therefore lengthened to $1.351 \AA$, while a standard double bond length is $1.322 \AA .^{6}$ Apparently, this is due to the steric hindrances around the double bond.

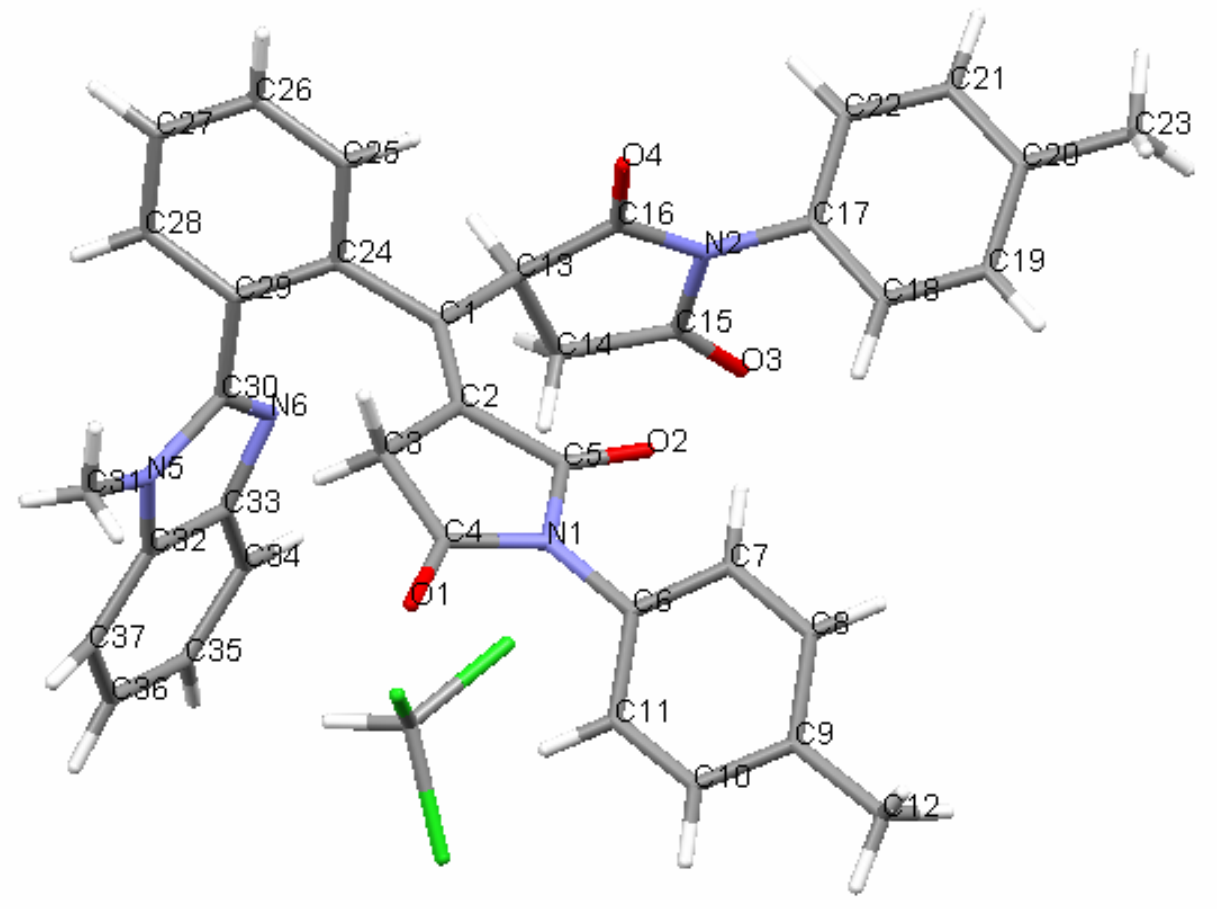

Figure 1. Mercury 1.4.2 sticks drawing of the crystal structure of $\mathbf{6 a}$.

To explain this unexpected reaction we propose below a probable mechanism. We postulate first a Michael- type reaction of the isoindole 2 with one equivalent of corresponding maleimide (3a-f), forming the succinimide derivative 4. The second step may be a Diels-Alder reaction of the intermediate 4 with the second equivalent of maleimide giving the 7-azanorbornene derivative 5. The latter rearranges in the isolated products $\mathbf{6 a - f}$ in one or more additional steps. This is associated to the destruction of two bonds in the strained cycle and the aromatization of the benzimidazole ring.

Interestingly, in spite of their great difference in electronic properties, similar products $\mathbf{8}$ were obtained in the reaction of pyrido[2,1-a] isoindole 7 with maleimides ${ }^{3 b, c}$ (Scheme 2). In fact, 7 is a $14 \pi$ - electron heteroaromatic system ${ }^{4 a, b}$ in contrast to the $10 \pi$ - electronic system of isoindolo[2,1-a]benzimidazole. But there is a weaker interaction between isoindole and the annulated benzimidazole fragments, ${ }^{4 \mathrm{c}}$ which makes it more similar to the $10 \pi$ - electronic system of the simple isoindoles. 


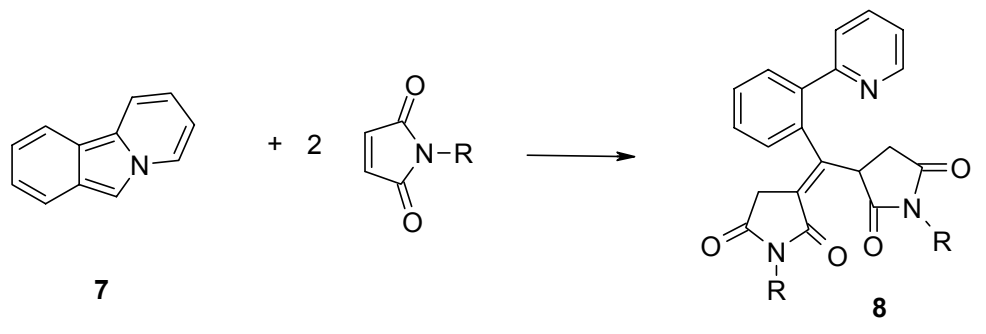

\section{Scheme 2}

Thus, the observation of Michael adducts for pyrido[2,1-a]isoindole, using GC-MS and GCUV spectra ${ }^{7}$ and the isolation of 7-azanorbornene derivatives $\mathbf{1 0}$ in the reaction of isoindolo[2,1a]quinazolin-5-one 9 with maleimide derivatives ${ }^{3 e}$ (Scheme 3) can be considered as supplementary arguments for the proposed mechanism.<smiles>Cn1c(=O)c2ccccc2n2cc3ccccc3c12</smiles>

9

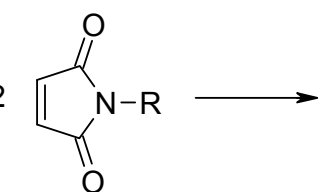

O

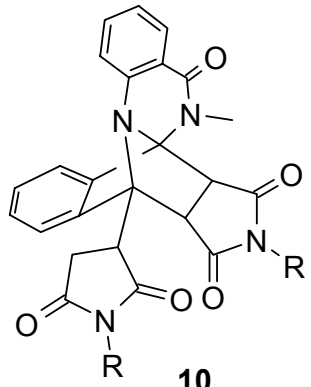

\section{Scheme 3}

\section{Spectroscopic properties of compounds 6a-f}

The ${ }^{1} \mathrm{H}$ NMR spectra of compounds $\mathbf{6 a}-\mathbf{f}$ present more signals than expected from the formulae. Moreover, there is only one spot in TLC. This is due to the presence of two chiral elements in the compounds (Scheme 4) - a chiral axis (in bold) and chiral center (marked by an asterisk), which may lead up to four isomeric forms. Furthermore, after treating the products $6 \mathbf{a}-\mathbf{f}$ with $\mathrm{D}_{2} \mathrm{O}$ we observe in the ${ }^{1} \mathrm{H}$ - NMR spectra the exchange of a broad singlet near $6 \mathrm{ppm}$, which indicates the presence of an enol form (Scheme 4) in the reaction mixture. Thus, in all cases, we observed the signals of three isomers - two atropodiastereomers and one enol form. All signals in ${ }^{1} \mathrm{H}$ - NMR spectra are assigned by 2D NMR and decoupling experiments. The spectra of the aliphatic part $(2.00-5.00 \mathrm{ppm})$ of all adducts are similar and this spectral range will be described here for compound 6a. 


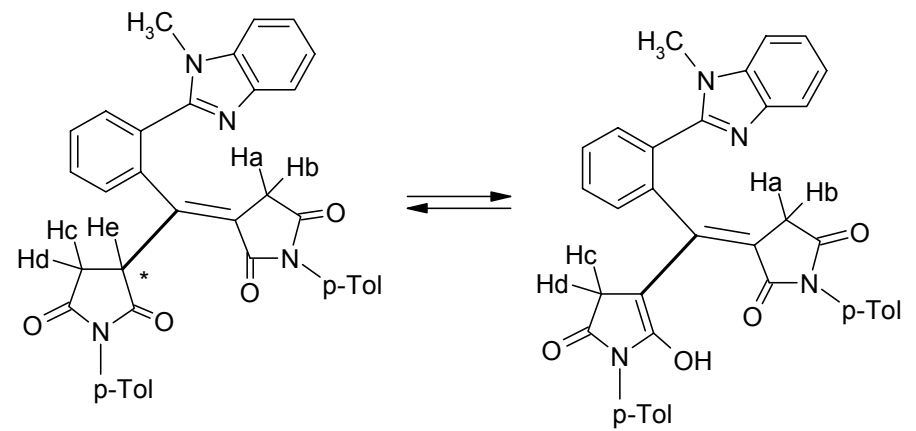

\section{Scheme 4}

The two diastereomers (I, II) and the enol (III) of 6a are in the ratio 100:35:59 respectively. We show the most characteristic fragment in Figure 2. The doublet of doublets of proton $\mathrm{H}_{\mathrm{c}}$ (II) is located at $2.60 \mathrm{ppm}$. The signal of the same proton $\mathrm{H}_{\mathrm{c}}$ of the isomer $\mathrm{I}\left(\mathrm{H}_{\mathrm{c}}(\mathrm{I})\right)$ is at weaker field at $2.97 \mathrm{ppm}$. The doublet for proton $\mathrm{H}_{\mathrm{a}}(\mathrm{I})(J=21.5 \mathrm{~Hz})$ is situated at $3.04 \mathrm{ppm}$. We observe the same value of coupling constant between $\mathrm{H}_{\mathrm{a}}-\mathrm{H}_{\mathrm{b}}$ protons for all isomers (I, II, III) of $\mathbf{6 a}-\mathbf{f}$. The doublets of doublets of protons $\mathrm{H}_{\mathrm{d}}$ of I and II isomers have similar chemical shifts (3.15 and 3.18 ppm respectively) and appears as a complicated multiplet. A doublet at $3.25 \mathrm{ppm}$ with $J 21.5 \mathrm{~Hz}$, mentioned before, correspond to the two $\mathrm{H}_{\mathrm{b}}$ protons $\left[\mathrm{H}_{\mathrm{b}}\right.$ (I) and $\mathrm{H}_{\mathrm{b}}$ (II)] according to their intensity. The next doublet at $3.38 \mathrm{ppm}$ has also a $J$ of $21.5 \mathrm{~Hz}$ and corresponds to the $\mathrm{H}_{\mathrm{a}}$ proton of isomer II. Obviously, the signals of $\mathrm{H}_{\mathrm{c}}$ and $\mathrm{H}_{\mathrm{d}}$ protons in enol III are doublets and not doublets of doublets as there is no $\mathrm{H}_{\mathrm{e}}$ proton in the enol form. Two such doublets are observed at 3.44 and $3.48 \mathrm{ppm}$. All ${ }^{1} \mathrm{H}$ NMR signals for compound $\mathbf{6 a}$ are summarized in the Table 1.

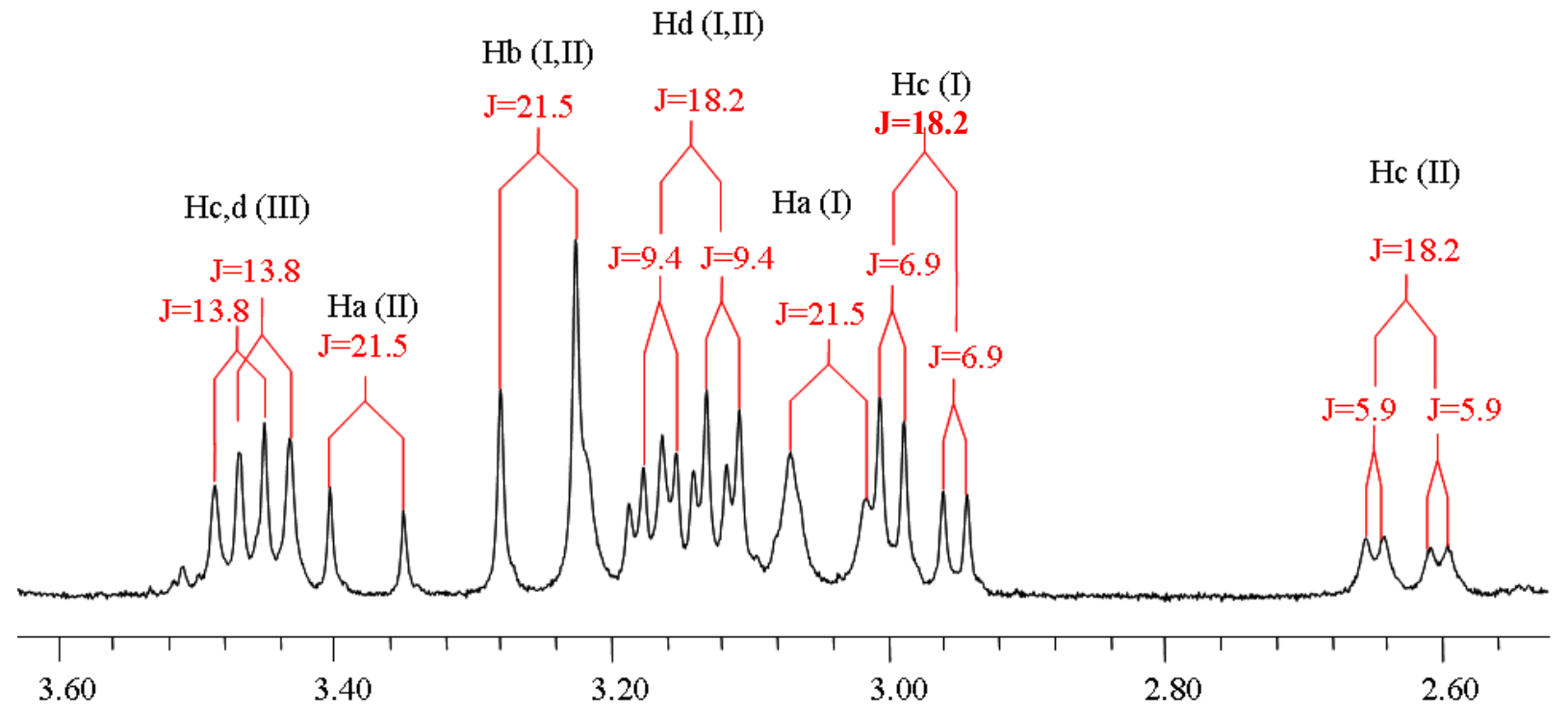

Figure 2. ${ }^{1} \mathrm{H}-400 \mathrm{MHz}$ spectrum of $\mathbf{6 a}$ in $\mathrm{CDCl}_{3}$ in the aliphatic range. 
Similar spectra were also observed for compounds $\mathbf{8}^{3 \mathrm{c}}$ with a pyridine instead of the benzimidazole ring. In this case, the existence of a diastereomeric mixture was proved using temperature-dependent NMR spectra ${ }^{3 \mathrm{c}}$ in which the coalescence of signals took place with increasing the temperature, thus lowering the rotation barrier about the chiral bond.

Table 1. Chemical shifts and coupling constants in ${ }^{1} \mathrm{H}$ NMR- spectra of compound $6 \mathbf{a}$

\begin{tabular}{llll}
\hline Proton & Isomer I, $\delta,(J, \mathrm{~Hz})$ & Isomer II, $\delta,(J, \mathrm{~Hz})$ & $\begin{array}{l}\text { Isomer III, } \delta,(J, \mathrm{~Hz}) \\
\text { Enol form }\end{array}$ \\
\hline $\mathrm{Ar}-\mathrm{CH}_{3}$ & $2.38(\mathrm{~s}) ; 2.39(\mathrm{~s})$ & $2.34(\mathrm{~s}) ; 2.43(\mathrm{~s})$ & $2.37(\mathrm{~s}) ; 2.40(\mathrm{~s})$ \\
$\mathrm{H}_{\mathrm{a}}$ & $3.04(\mathrm{~d}, 21.5)$ & $3.38(\mathrm{~d}, 21.5)$ & $3.95(\mathrm{~d}, 21.5)$ \\
$\mathrm{H}_{\mathrm{b}}$ & $3.25(\mathrm{~d}, 21.5)$ & $3.25(\mathrm{~d}, 21.5)$ & $3.70(\mathrm{~d}, 21.5)$ \\
$\mathrm{H}_{\mathrm{c}}$ & $2.97(\mathrm{dd}, 6.7 ; 18.2)$ & $2.62(\mathrm{dd}, 5.9 ; 18.2)$ & $3.44(\mathrm{~d}, 13.8)$ \\
$\mathrm{H}_{\mathrm{d}}$ & $3.15(\mathrm{dd}, 9.5 ; 18.2)$ & $3.18(\mathrm{dd}, 9.5 ; 18.2)$ & $3.48(\mathrm{~d}, 13.8)$ \\
$\mathrm{H}_{\mathrm{e}}$ & $4.20(\mathrm{~m}) ;$ & $4.30(\mathrm{t}, 7.0) ;$ & $5.67(\mathrm{br} \mathrm{s})$ \\
$\mathrm{N}-\mathrm{CH}_{3}$ & $3.87(\mathrm{~s})$ & $3.81(\mathrm{~s})$ & $3.67(\mathrm{~s})$ \\
$\mathrm{H}_{\mathrm{Ar}}$ & & $6.94-7.81(\mathrm{~m})$ & \\
\hline
\end{tabular}

\section{Conclusions}

The reaction of 5-methyl-5H-isoindolo[2,1-a]benzimidazole with maleimide derivatives leads to rearranged products $\mathbf{6 a - f}$ in good yields. The proposed mechanism of the reaction begins by a Michael addition of one equivalent of maleimide to the isoindole followed by a Diels-Alder cycloaddition of the second equivalent of maleimide and subsequent rearrangement of the intermediate tricyclic 7-azanorbornene derivatives 5a-f. From X-ray structure analysis of compound 6a, it appears that the $\mathrm{C}-\mathrm{C}$ - double bond is twisted (torsion angle $6.42^{\circ}$ ). A detailed analysis of NMR- spectra of compounds $\mathbf{6 a}-\mathbf{f}$ shows the presence in solution of a mixture of two diastereomers and one enol form.

\section{Experimental Section}

General Procedures. Uncorrected melting points were measured by a Boetius-Thiele apparatus. IR spectra were recorded on a Perkin-Elmer 1760X FT-IR, the EI mass-spectra (70 eV) on a Nermag R10 at the "Service Commun de Spectrométrie de Masse" of the Paul Sabatier University Toulouse, and the ${ }^{13} \mathrm{C}$ - and other NMR spectra on a Varian Mercury $400(400 \mathrm{MHz})$ $\left({ }^{1} \mathrm{H}, 400 \mathrm{MHz},{ }^{13} \mathrm{C}, 100 \mathrm{MHz}\right)$ in $\mathrm{CDCl}_{3}, \delta$ values are given in ppm with TMS as internal standard. Elemental analysis were performed at the Department of Chemistry, Kyiv National Taras Shevchenko University. 
3-(E)-1-(2,5-Dioxo-1- $R$-tetrahydro-1H-3-pyrrolyl)-1-[2-(1-methyl-1H-benzo[ $d]$ imidazol-2-


of $1 \mathrm{mmol}(0.35 \mathrm{~g}) 5$-methyl-5H-isoindolo[2,1-a]benzimidazolium iodide 1 (obtained according to the literature $\left.{ }^{8}\right)$ and $2 \mathrm{mmol}$ of the corresponding maleimide (3a-f) was added $2.5 \mathrm{mmol}(0.25$ g) of triethylamine. This solution was heated at reflux for $4 \mathrm{~h}$ and then evaporated in vacuo. The residue was purified by column chromatography $\left(\mathrm{CH}_{2} \mathrm{Cl}_{2} / \mathrm{MeOH}, 100: 1\right)$.

3-(E)-1-[2-(1-Methyl-1H-benzo[d]imidazol-2-yl)phenyl]-1-[1-(4-methylphenyl)-2,5dioxotetrahydro-1H-3-pyrrolyl]methylidene-1-(4-methylphenyl)-2,5-pyrrolidinedione (6a). Treatment of $0.35 \mathrm{~g}$ ( $1 \mathrm{mmol}) 5$-methyl-5H-isoindolo[2,1-a]benzimidazolium iodide 1 and 0.37 $\mathrm{g}(2 \mathrm{mmol}) \mathrm{N}$-(p-tolyl)maleimide 3a according to the general procedure gave $0.42 \mathrm{~g}(0.71 \mathrm{mmol}$, 71\%) 6a as brown solid, mp: 139-141 ${ }^{\circ} \mathrm{C}$. IR (KBr): 3035 (w), $2950(\mathrm{w}), 2922$ (m), $2866(\mathrm{~m})$, $1710(\mathrm{~s}), 1514$ (s), 1473 (m), 1456 (m), 1429 (m), 1380 (s), 1326 (m), 1282 (m), 1195 (s), 1141 (s), $1053(\mathrm{~m}), 1022(\mathrm{~m}), 1004(\mathrm{~m}), 939(\mathrm{w}), 912(\mathrm{~m}), 815$ (m), 769 (m), 759 (m), $746(\mathrm{~m}), 715$ (m), $648(\mathrm{~m}), 584(\mathrm{~m}), 509(\mathrm{~m}) \mathrm{cm}^{-1}$.

${ }^{1} \mathrm{H}$ NMR (400 MHz, $\mathrm{CDCl}_{3}$ ): 2.34 (s, Ar-CH3 (II)), 2.37 (s, Ar-CH3 (III)), 2.38 (s, Ar-CH (I)), 2.39 (s, $\left.\mathrm{Ar}_{-} \mathrm{CH}_{3}(\mathrm{I})\right), 2.40$ (s, $\left.\mathrm{Ar}_{-} \mathrm{CH}_{3}(\mathrm{III})\right), 2.43$ (s, Ar-CH3 (II)), 2.62 (dd, $J=5.9,18.2 \mathrm{~Hz}, H_{\mathrm{c}}$ (II)), 2.97 (dd, $\left.J=6.7,18.2 \mathrm{~Hz}, H_{\mathrm{c}}(\mathrm{I})\right), 3.04$ (d, $\left.J=21.5 \mathrm{~Hz}, H_{\mathrm{a}}(\mathrm{I})\right), 3.15$ (dd, $J=9.5,18.2 \mathrm{~Hz}$, $\left.\mathrm{H}_{\mathrm{d}}(\mathrm{I})\right), 3.18$ (dd, $\left.J=9.5,18.2 \mathrm{~Hz}, \mathrm{H}_{\mathrm{d}}(\mathrm{II})\right), 3.25$ (d, $\left.J=21.5 \mathrm{~Hz}, \mathrm{H}_{\mathrm{b}}(\mathrm{I}, \mathrm{II})\right), 3.38$ (d, $J=21.5 \mathrm{~Hz}$, $\left.\mathrm{H}_{\mathrm{a}}(\mathrm{II})\right), 3.44$ (d, $J=13.8 \mathrm{~Hz}, \mathrm{H}_{\mathrm{c}}$ (III)), 3.48 (d, $J=13.8 \mathrm{~Hz}, \mathrm{H}_{\mathrm{d}}$ (III)), 3.67 (s, N-CH $\left.(\mathrm{III})\right), 3.70$ (d, $\left.J=21.5 \mathrm{~Hz}, \mathrm{H}_{\mathrm{b}}(\mathrm{III})\right), 3.81$ (s, N-CH $\left.(\mathrm{II})\right), 3.87$ (s, N-CH$\left.(\mathrm{I})\right), 3.95$ (d, $J=21.5 \mathrm{~Hz}, H_{\mathrm{a}}$ (III)), $4.20\left(\mathrm{~m}, H_{\mathrm{e}}(\mathrm{I})\right), 4.30\left(\mathrm{t}, J=7.0 \mathrm{~Hz}, H_{\mathrm{e}}(\mathrm{II})\right), 5.67$ (br s, $\left.H_{\mathrm{e}}(\mathrm{III})\right), 6.94-7.81\left(\mathrm{~m}, \mathrm{H}_{\text {arom}}\right)$ ppm. Ratio I:II:III = 100:35:59. ${ }^{13} \mathrm{C}$ NMR $\left(100 \mathrm{MHz}, \mathrm{CDCl}_{3}\right): 21.21,31.45,34.11,34.71,34.20,34.44$, $37.43,47.84,61.85,109.69,109.85,109.94$. 119.80, 120.16, 120.28, 122.68, 122.93, 123.35 , $123.45,126.16,126.20,126.84,128.17,128.52$, 129.22, 129.61, 129.76, 129.84, 129.96, 130.42, $130.90,131.14,131.16,135.90,138.55,138.67,142.76,148.84,151.11,172.25,172.35,175.78$, 175.97 ppm. MS (EI, $70 \mathrm{eV}): \mathrm{m} / \mathrm{z}(\%)=594$ (16.28) [M] ${ }^{+}, 432$ (15.49), 406 (100.00), 299 (3.77), 246 (25.37), 133 (7.05), 107 (11.66), 55 (4.91). Anal. Calcd. for $\mathrm{C}_{37} \mathrm{H}_{30} \mathrm{~N}_{4} \mathrm{O}_{4}: \mathrm{C} 74.73, \mathrm{H} \mathrm{5.08,} \mathrm{N}$ 9.42. Found C 74.80, H 5.12, N 9.50.

\section{X-Ray crystal structure analysis of $6 \mathbf{a}$}

The X-ray structure determination was performed at room temperature $(298 \mathrm{~K})$ for $\mathbf{2 a}$ on a Nonius Kappa CCD diffractometer with a graphite oriented monochromator utilizing $\mathrm{MoK}_{\alpha}$ radiation $(\lambda=0.71073)$. The structure was solved by direct methods and refined by least-squares procedures. All diagrams and Calculations were performed using maXus package programs (Mac Science, Japan). The crystals of compound 6a are triclinic $\left(\mathrm{C}_{37} \mathrm{H}_{30} \mathrm{~N}_{4} \mathrm{O}_{4} ; \mathrm{CHCl}_{3}\right)$. $a=10.947(2) \AA, b=11.5051(8) \AA, c=15.376(5) \AA, \alpha=70.40(2)^{\circ}, \beta=77.77(3)^{\circ}, \gamma=82.932(15)^{\circ}$, $\mathrm{V}=1780.1(7) \AA{ }^{3}, \mathrm{~d}=1.332 \mathrm{~g} / \mathrm{cm}^{3}$, space group $\mathrm{P} 1$ (bar), $\mathrm{Z}=2$. Intensities of 2776 unique reflections $\left(\mathrm{R}=0.064 ; \mathrm{R}_{\mathrm{W}}=0.121\right)$ were measured. 
The crystallographic data submission CCDC 637008 contains the supplementary crystallographic data for 6a. These data can be obtained free of charge from The Cambridge Crystallographic Data Centre via www.ccdc.cam.ac.uk/data_request/cif.

\section{1-(4-Methoxyphenyl)-3-(E)-1-[1-(4-methoxyphenyl)-2,5-dioxotetrahydro-1H-3-pyrrolyl]-1-} [2-(1-methyl-1H-benzo[d]imidazol-2-yl)phenyl]methylidene-2,5-pyrrolidinedione

(6b).

Compound $\mathbf{6 b}$ was obtained from $0.35 \mathrm{~g} \quad(1 \mathrm{mmol})$ 5-methyl-5H-isoindolo[2,1a]benzimidazolium iodide $\mathbf{1}$ and $0.41 \mathrm{~g}$ ( $2 \mathrm{mmol}) N$-( $p$-anisyl)maleimide $\mathbf{3 b}$ according to the general procedure in $85 \%$ yield $(0.50 \mathrm{~g}, 0.85 \mathrm{mmol})$ as a brown solid, $\mathrm{mp}: 137-138^{\circ} \mathrm{C}$. $\mathrm{IR}(\mathrm{KBr})$ : 3055 (w), 2937 (m), 2837 (m), 1708 (s), 1660 (m), 1610 (m), 1591 (m), 1512 (s), 1461 (m), 1440 (m), $1429(\mathrm{~m}), 1382(\mathrm{~s}), 1328(\mathrm{~m}), 1299(\mathrm{~m}), 1284(\mathrm{~m}), 1274(\mathrm{~m}), 1249$ (s), 1195 (s), 1180 (s), 1168 (s), 1143 (s), 1107 (m), 1053 (m), 1028 (m), 931 (m), 912 (m), 831 (m), 810 (m), 767 (m), $746(\mathrm{~s}), 702(\mathrm{~m}), 688(\mathrm{~m}), 663(\mathrm{~m}), 640(\mathrm{~m}), 594(\mathrm{~m}), 526(\mathrm{~m}) \mathrm{cm}^{-1} .{ }^{1} \mathrm{H}$ NMR (400 MHz, $\left.\mathrm{CDCl}_{3}\right): 2.61\left(\mathrm{dd}, J=5.0,18.3 \mathrm{~Hz}, H_{\mathrm{c}}(\mathrm{II})\right), 2.94\left(\mathrm{dd}, J=6.9,18.3 \mathrm{~Hz}, H_{\mathrm{c}}(\mathrm{I})\right), 2.98(\mathrm{~d}, J=21.5$ $\left.\mathrm{Hz}, H_{\mathrm{a}}(\mathrm{I})\right), 3.16\left(\mathrm{~d}, J=8.5 \mathrm{~Hz}, H_{\mathrm{d}}(\mathrm{I})^{*}\right), 3.10\left(\mathrm{~d}, J=9.4 \mathrm{~Hz}, H_{\mathrm{d}}(\mathrm{II})^{*}\right), 3.21$ (d, $J=21.5 \mathrm{~Hz}, H_{\mathrm{b}}$ (I, II)), 3.33 (d, $\left.J=21.5 \mathrm{~Hz}, H_{\mathrm{a}}(\mathrm{II})\right), 3.39$ (d, $\left.J=13.7 \mathrm{~Hz}, H_{\mathrm{c}}(\mathrm{III})\right), 3.43$ (d, $J=13.7 \mathrm{~Hz}, H_{\mathrm{d}}$ (III)), 3.69 (d, $\left.J=21.5 \mathrm{~Hz}, H_{\mathrm{b}}(\mathrm{III})\right), 3.73\left(\mathrm{~d}, J=21.5 \mathrm{~Hz}, H_{\mathrm{a}}(\mathrm{III})\right), 3.76\left(\mathrm{CH}_{3}\right), 3.77\left(\mathrm{CH}_{3}\right), 3.78$ $\left(\mathrm{CH}_{3}\right), 3.79\left(\mathrm{CH}_{3}\right), 3.80\left(\mathrm{CH}_{3}\right), 3.81\left(\mathrm{CH}_{3}\right), 3.811\left(\mathrm{CH}_{3}\right), 3.83\left(\mathrm{CH}_{3}\right), 3.84\left(\mathrm{CH}_{3}\right), 4.17(\mathrm{t}, J=8.0$ $\left.\mathrm{Hz}, H_{\mathrm{e}}(\mathrm{I})\right), 4.27$ (t, $\left.J=7.2 \mathrm{~Hz}, H_{\mathrm{e}}(\mathrm{II})\right), 5.69$ (br s, $\left.H_{\mathrm{e}}(\mathrm{III})\right), 6.87-7.84$ (m, $\left.H_{\text {arom }}\right)$ ppm. Ratio I:II:III = 100:29:62; III is the enol form. ${ }^{13} \mathrm{C} \mathrm{NMR}\left(100 \mathrm{MHz}, \mathrm{CDCl}_{3}\right): 24.53,31.43,34.06$, $35.15,37.41,43.82,55.48,109.36,109.85,110.33,114.27,114.39,114.44,114.61,120.18$, $120.30,122.72,122.96,123.38,123.48,125.14,127.60,127.64,128.18,128.26,128.81,128.96$, $129.22,129.96,130.44,130.60,130.95,135.88,135.97,142.77,159.43,159.52,167.78,172.36$, 172.45, 175.96, $176.13 \mathrm{ppm}$. MS (EI, $70 \mathrm{eV}): \mathrm{m} / \mathrm{z}(\%)=626(60.10)[\mathrm{M}]^{+}, 476(6.25), 422$ (100.00), 4.06 (5.73), 299 (6.13), 246 (26.26), 205 (6.68), 123 (13.01), 108 (10.31), 55 (3.15). Anal. Calcd. for $\mathrm{C}_{37} \mathrm{H}_{30} \mathrm{~N}_{4} \mathrm{O}_{6}$ : C 70.92, H 4.83, N 8.94. Found C 71.20, H 5.01, N 9.10.

\section{1-(4-Bromophenyl)-3-(E)-1-[1-(4-bromophenyl)-2,5-dioxotetrahydro-1H-3-pyrrolyl]-1-[2-} (1-methyl-1H-benzo[d]imidazol-2-yl)phenyl]methylidene-2,5-pyrrolidinedione

(6c).

Treatment of $0.35 \mathrm{~g}$ (1 mmol) 5-methyl-5H-isoindolo[2,1- $a$ ] benzimidazolium iodide 1 and 0.50 g (2 mmol) $N$-(p-bromophenyl)maleimide 3c gave $6 \mathbf{c}(0.58 \mathrm{~g}, 0.80 \mathrm{mmol}, 80 \%)$ as a brown solid, mp: $149-150^{\circ} \mathrm{C}$. IR (KBr): $3091(\mathrm{~m}), 3057(\mathrm{w}), 2947$ (w), 1710 (s), $1654(\mathrm{~m}), 1490$ (s), 1458 (m), 1429 (m), 1379 (s), 1328 (m), 1280 (m), 1193 (m), 1178 (m), 1139 (m), 1097 (m), 1070 (m), $1031(\mathrm{w}), 1012(\mathrm{~m}), 937(\mathrm{w}), 910(\mathrm{~m}), 823(\mathrm{~m}), 800(\mathrm{~m}), 773(\mathrm{~m}), 765(\mathrm{~m}), 746(\mathrm{~m}), 711$ (m), 669 (w), 649 (m), $634(\mathrm{~m}), 543(\mathrm{w}), 505(\mathrm{~m}) \mathrm{cm}^{-1} .{ }^{1} \mathrm{H}$ NMR (400 MHz, $\left.\mathrm{CDCl}_{3}\right): 2.68$ (dd, $J$ $\left.=5.2,18.3 \mathrm{~Hz}, H_{\mathrm{c}}(\mathrm{II})\right), 2.94\left(\mathrm{dd}, J=6.8,17.3 \mathrm{~Hz}, H_{\mathrm{c}}(\mathrm{I})\right), 2.97\left(\mathrm{~d}, J=21.5 \mathrm{~Hz}, H_{\mathrm{a}}(\mathrm{I})\right), 3.14(\mathrm{dd}$, $\left.J=9.5,18.3 \mathrm{~Hz}, H_{\mathrm{d}}(\mathrm{I})\right), 3.19\left(\mathrm{dd}, J=7.1,17.3 \mathrm{~Hz}, H_{\mathrm{d}}(\mathrm{II})\right), 3.20\left(\mathrm{~d}, J=21.5 \mathrm{~Hz}, H_{\mathrm{b}}\right.$ (I, II)), 3.34 (d, $\left.J=21.5 \mathrm{~Hz}, H_{\mathrm{a}}(\mathrm{II})\right), 3.39$ (d, $\left.J=12.7 \mathrm{~Hz}, H_{\mathrm{c}}(\mathrm{III})\right), 3.41$ (d, $\left.J=12.7 \mathrm{~Hz}, H_{\mathrm{d}}(\mathrm{III})\right), 3.48$ (d, $J$ $\left.=21.5 \mathrm{~Hz}, H_{\mathrm{b}}(\mathrm{III})\right), 3.50\left(\mathrm{~d}, J=21.5 \mathrm{~Hz}, H_{\mathrm{a}}(\mathrm{III})\right), 3.65\left(\mathrm{~N}-\mathrm{CH}_{3}(\mathrm{III})\right), 3.77\left(\mathrm{~N}-\mathrm{CH} H_{3}(\mathrm{II})\right), 3.85$ $\left(\mathrm{N}-\mathrm{CH}_{3}(\mathrm{I})\right), 4.18\left(\mathrm{t}, J=8.0 \mathrm{~Hz}, H_{\mathrm{e}}(\mathrm{I})\right), 4.28$ (t, $\left.J=8.0 \mathrm{~Hz}, H_{\mathrm{e}}(\mathrm{II})\right), 5.59$ (br s, $\left.H_{\mathrm{e}}(\mathrm{III})\right), 6.89-$ 8.10 (m, $\left.H_{\text {arom}}\right)$ ppm. Ratio I:II:III $=100: 52: 30$; III is the enol form. ${ }^{13} \mathrm{C}$ NMR $(100 \mathrm{MHz}$, $\left.\mathrm{CDCl}_{3}\right): 23.31,31.46,35.16,37.48,44.17,47.89,53.47,109.72,109.91,120.17,120.31,122.31$, 
$122.50,122.82,122.06,123.52,123.61,127.85,127.89,127.95,128.05,128.10,128.28,128.57$, $128.70,130.28,130.45,130.64,130.99,131.25,132.12,132.25,132.26,132.31,132.51,135.98$, 142.82, 167.34, 171.62, 175.14, 175.38, $176.62 \mathrm{ppm} . \mathrm{MS}(\mathrm{EI}, 70 \mathrm{eV}): \mathrm{m} / \mathrm{z}(\%)=723(20.63)$ $[\mathrm{M}]^{+}, 571$ (0.65), 470 (100.00), 391 (4.52), 271 (20.90), 246 (24.83), 171 (7.96), 113 (28.15), 55 (10.05). Anal. Calcd. for $\mathrm{C}_{35} \mathrm{H}_{24} \mathrm{Br}_{2} \mathrm{~N}_{4} \mathrm{O}_{4}$ : C 58.03, $\mathrm{H} 3.34, \mathrm{~N} 7.73$. Found $\mathrm{C} 58.20, \mathrm{H} \mathrm{3.42,} \mathrm{N}$ $7.94 \%$.

\section{1-(4-Chlorophenyl)-3-(E)-1-[1-(4-chlorophenyl)-2,5-dioxotetrahydro-1H-3-pyrrolyl]-1-[2-} (1-methyl-1H-benzo[d]imidazol-2-yl)phenyl]methylidene-2,5-pyrrolidinedione

(6d).

Treatment of 5-methyl-5H-isoindolo[2,1-a] benzimidazolium iodide 1 (0.35 g, $1 \mathrm{mmol})$ and 0.42 g $(2 \mathrm{mmol})$ of $N$-( $p$-chlorophenyl)maleimide 3d produced $6 \mathbf{d}(0.44 \mathrm{~g}, 0.69 \mathrm{mmol}, 69 \%)$ as a brown solid, mp 130-132 ${ }^{\circ} \mathrm{C}$. IR (KBr): 3095 (w), 3057 (w), 2979 (w), 2947 (w), 1712 (s), 1651 (m), $1614(\mathrm{~m}), 1595(\mathrm{~m}), 1531(\mathrm{~m}), 1492(\mathrm{~s}), 1458(\mathrm{~m}), 1429(\mathrm{~m}), 1379(\mathrm{~s}), 1326(\mathrm{~m}), 1282(\mathrm{~m})$, 1244 (m), 1191 (s), 1141 (s), 1089 (s), 1055 (m), 1031 (m), 1016 (s), 937 (m), 912 (m), 825 (s), 765 (s), 746 (s), $713(\mathrm{~m}), 649(\mathrm{~m}), 507(\mathrm{~m}), 470(\mathrm{w}), 418(\mathrm{~m}) \mathrm{cm}^{-1} .{ }^{1} \mathrm{H}$ NMR (400 MHz, $\left.\mathrm{CDCl}_{3}\right)$ : $2.69\left(\mathrm{dd}, J=5.6,18.2 \mathrm{~Hz}, H_{\mathrm{c}}(\mathrm{II})\right), 2.95\left(\mathrm{dd}, J=7.3,18.7 \mathrm{~Hz}, H_{\mathrm{c}}(\mathrm{I})\right), 2.96\left(\mathrm{~d}, J=21.5 \mathrm{~Hz}, H_{\mathrm{a}}\right.$ (I)), $3.14\left(\mathrm{~d}, J=9.6 \mathrm{~Hz}, H_{\mathrm{d}}(\mathrm{I})^{*}\right), 3.21$ (d, $\left.J=9.8 \mathrm{~Hz}, H_{\mathrm{d}}(\mathrm{II})^{*}\right), 3.22$ (d, $\left.J=21.5 \mathrm{~Hz}, H_{\mathrm{b}}(\mathrm{I}, \mathrm{II})\right)$, $3.34\left(\mathrm{~d}, J=21.5 \mathrm{~Hz}, \mathrm{H}_{\mathrm{a}}(\mathrm{II})\right), 3.40\left(\mathrm{~m}, H_{\mathrm{c}}, H_{\mathrm{d}}(\mathrm{III})\right), 3.49$ (d, $\left.J=21.5 \mathrm{~Hz}, H_{\mathrm{b}}(\mathrm{III})\right), 3.60$ (d, $J=$ $\left.21.5 \mathrm{~Hz}, H_{\mathrm{a}}(\mathrm{III})\right), 3.70\left(\mathrm{~N}-\mathrm{CH}_{3}(\mathrm{III})\right), 3.78\left(\mathrm{~N}-\mathrm{CH}_{3}(\mathrm{II})\right), 3.85\left(\mathrm{~N}-\mathrm{CH}_{3}(\mathrm{I})\right), 4.18\left(\mathrm{~m}, H_{\mathrm{e}}(\mathrm{I}, \mathrm{II})\right)$, $5.59\left(\right.$ br s, $\left.H_{\mathrm{e}}(\mathrm{III})\right), 6.78-8.06\left(\mathrm{~m}, H_{\text {arom}}\right)$ ppm. Ratio I:II:III $=100: 58: 30 .{ }^{13} \mathrm{C} \mathrm{NMR}(100 \mathrm{MHz}$, $\left.\mathrm{CDCl}_{3}\right): 18.30,31.49,35.12,37.48,47.30,47.84,53.43,109.40,109.76,109.88,109.98,120.12$, $120.23,120.52,122.77,123.02,123,47,123.58,123.87,127.20,127.55,127.65,127.75,128.05$, $128.26,128.69,128.83,129.13,129.22,129.26,129.32,129.60,129.71,130.60,130.98,134.39$, 166.42, 170.25, 171.68, 175.30 ppm. MS (EI, $70 \mathrm{eV}): \mathrm{m} / \mathrm{z}(\%)=634(12.75)[\mathrm{M}]^{+}, 526(4.48)$, 426 (100.00), 299 (4.21), 246 (29.03), 206 (8.11), 127 (25.43), 113 (17.03), 55 (5.97). Anal. Calcd. for $\mathrm{C}_{35} \mathrm{H}_{24} \mathrm{Cl}_{2} \mathrm{~N}_{4} \mathrm{O}_{4}$ : C 66.15, $\mathrm{H}$ 3.81, N 8.82. Found C 66.25, H 4.06, N 8.96.

\section{3-(E)-1-[2-(1-Methyl-1H-benzo[d]imidazol-2-yl)phenyl]-1-[1-(3-nitrophenyl)-2,5-dioxo-} tetrahydro-1H-3-pyrrolyl]methylidene-1-(3-nitrophenyl)-2,5-pyrrolidinedione

(6e).

Treatment of 5-methyl-5H-isoindolo[2,1-a]benzimidazolium iodide 1, $(0.35 \mathrm{~g}, 1 \mathrm{mmol})$ and $\mathrm{N}-(\mathrm{m}$ -nitrophenyl)maleimide 3e $(0.44 \mathrm{~g}, 2 \mathrm{mmol})$ gave $6 \mathrm{e}(0.47 \mathrm{~g}, 0.72 \mathrm{mmol}, 72 \%)$ as a brown solid, mp 128-130 C. IR (KBr): 3089 (w), 3057 (w), 2976 (w), 2945 (w), 2869 (w), 1714 (s), 1614 (m), 1600 (m), 1531 (s), 1481 (m), 1461 (m), 1431 (m), 1379 (s), 1350 (s), 1296 (m), 1284 (m), 1245 (m), 1197 (s), 1178 (s), 1093 (m), 1056 (m), 1029 (m), 1004 (m), 948 (m), 931 (w), 889 (m), $858(\mathrm{w}), 821(\mathrm{~m}), 802(\mathrm{~m}), 767(\mathrm{~m}), 738(\mathrm{~s}), 675(\mathrm{~m}), 642(\mathrm{~m}), 619(\mathrm{~m}), 595(\mathrm{~m}), 578(\mathrm{~m})$, $543(\mathrm{w}), 522(\mathrm{w}), 516(\mathrm{w}), 484(\mathrm{~m}) \mathrm{cm}^{-1} .{ }^{1} \mathrm{H}$ NMR (400 MHz, $\left.\mathrm{CDCl}_{3}\right): 2.87$ (dd, $J=6.0,14.0$ $\left.\mathrm{Hz}, H_{\mathrm{c}}(\mathrm{II})\right), 3.01$ (dd, $\left.J=6.9,18.3 \mathrm{~Hz}, H_{\mathrm{c}}(\mathrm{I})\right), 3.02$ (d, $\left.J=21.5 \mathrm{~Hz}, H_{\mathrm{a}}(\mathrm{I})\right), 3.23$ (d, $J=10.1 \mathrm{~Hz}$, $\left.H_{\mathrm{d}}(\mathrm{I})^{*}\right), 3.29$ (d, $\left.J=10.1 \mathrm{~Hz}, H_{\mathrm{d}}(\mathrm{II})^{*}\right), 3.35$ (d, $\left.J=21.5 \mathrm{~Hz}, H_{\mathrm{b}}(\mathrm{I}, \mathrm{II})\right), 3.41$ (d, $J=21.5 \mathrm{~Hz}, H_{\mathrm{a}}$ (II)), $3.50\left(\mathrm{~m}, H_{\mathrm{c}}(\mathrm{III})\right), 3.46\left(\mathrm{~m}, H_{\mathrm{d}}(\mathrm{III})\right), 3.58$ (d, $\left.J=21.5 \mathrm{~Hz}, H_{\mathrm{b}}(\mathrm{III})\right), 3.69$ (d, $J=21.5 \mathrm{~Hz}, H_{\mathrm{a}}$ (III)), $3.72\left(\mathrm{~N}-\mathrm{CH}_{3}(\mathrm{I})\right), 3.84\left(\mathrm{~N}-\mathrm{CH}_{3}(\mathrm{III})\right), 3.90\left(\mathrm{~N}-\mathrm{CH}_{3}(\mathrm{II})\right), 4.21\left(\mathrm{~m}, H_{\mathrm{e}}(\mathrm{II})\right), 4.26\left(\mathrm{~m}, H_{\mathrm{e}}\right.$ (I)), 5.48 (br s, $\left.H_{\mathrm{e}}(\mathrm{III})\right), 7.18-8.29$ (m, $\left.H_{\text {arom }}\right)$ ppm. Ratio I:II:III $=100: 54: 30 .{ }^{13} \mathrm{C}$ NMR $(100$ $\left.\mathrm{MHz}, \mathrm{CDCl}_{3}\right): 20.24,31.24,31.53,34.12,109.44,109.85,109.99,118.63,120.77,120.46$, 
$121.52,122.27,122.84,123.08,123.31,123.42,123.93,128.71,128.77,129.83,130.07,140.26$, $131.72,132.13,132.27,133.93,148.35,165.94,168.77,171.22,172.18,174.87$ ppm. MS (EI, 70 $\mathrm{eV}): \mathrm{m} / \mathrm{z}(\%)=656$ (4.92) $[\mathrm{M}]^{+}, 437$ (32.48), 391 (4.10), 319 (12.48), 246 (62.60), 218 (30.12), 138 (100.00), 92 (87.99), 54 (12.67). Anal. Calcd. for $\mathrm{C}_{35} \mathrm{H}_{24} \mathrm{~N}_{6} \mathrm{O}_{8}$ : C 64.02, H 3.68, N 12.80. Found C 64.30, H 3.86, N 13.14\%.

3-(E)-1-(2,5-Dioxo-1-phenyltetrahydro-1H-3-pyrrolyl)-1-[2-(1-methyl-1H-benzo[d]imidazol-2-yl)phenyl]methylidene-1-phenyl-2,5-pyrrolidinedione (6f). Treatment of 5methyl-5H-isoindolo[2,1-a]benzimidazolium iodide $1(0.35 \mathrm{~g}, 1 \mathrm{mmol})$ and $N$-phenyl-maleimide 3f $(0.35 \mathrm{~g}, 2 \mathrm{mmol})$ gave $6 \mathbf{f}(0.48 \mathrm{~g}, 0.85 \mathrm{mmol}, 85 \%)$ as a brown solid, $\mathrm{mp} 135-136^{\circ} \mathrm{C}$. IR (KBr): 3060 (w), $3001(\mathrm{w}), 2947$ (w), 1708 (s), 1656 (m), 1597 (m), 1560 (w), 1543 (w), 1535 (w), 1498 (s), 1456 (m), 1438 (m), 1429 (m), 1379 (s), 1326 (m), 1282 (m), 1265 (m), 1244 (m), 1191 (s), 1141 (s), 1099 (m), 1072 (m), 1053 (m), 1029 (m), 1004 (m), 937 (m), 910 (m), 823 (m), 804 (m), 746 (s), 694 (s), 669 (m), 644 (m), 619 (m), 541 (m), 503 (m). ${ }^{1} \mathrm{H}$ NMR (400 MHz, $\left.\mathrm{CDCl}_{3}\right): 2.63\left(\mathrm{dd}, J=5.4,18.9 \mathrm{~Hz}, H_{\mathrm{c}}(\mathrm{II})\right), 2.97$ (dd, $\left.J=7.1,18.4 \mathrm{~Hz}, H_{\mathrm{c}}(\mathrm{I})\right), 3.00$ (d, $J=21.5$ $\left.\mathrm{Hz}, H_{\mathrm{a}}(\mathrm{I})\right), 3.11\left(\mathrm{~d}, J=9.6 \mathrm{~Hz}, H_{\mathrm{d}}(\mathrm{I})^{*}\right), 3.17$ (d, $\left.J=10.5 \mathrm{~Hz}, H_{\mathrm{d}}(\mathrm{II})^{*}\right), 3.23$ (d, J=21.5 Hz, $H_{\mathrm{b}}$ (I, II)), 3.35 (d, $\left.J=21.5 \mathrm{~Hz}, H_{\mathrm{a}}(\mathrm{II})\right), 3.43$ (d, $\left.J=11.0 \mathrm{~Hz}, H_{\mathrm{c}}(\mathrm{III})\right), 3.47$ (d, $J=11.0 \mathrm{~Hz}, H_{\mathrm{d}}$ (III)), 3.54 (d, $\left.J=21.5 \mathrm{~Hz}, H_{\mathrm{b}}(\mathrm{III})\right), 3.58$ (d, $\left.J=21.5 \mathrm{~Hz}, H_{\mathrm{a}}(\mathrm{III})\right), 3.64$ (N-CH3 (III)), 3.77 (N$\left.\mathrm{CH}_{3}(\mathrm{II})\right), 3.83\left(\mathrm{~N}-\mathrm{CH}_{3}(\mathrm{I})\right), 4.20\left(\mathrm{~m}, H_{\mathrm{e}}(\mathrm{I})\right), 4.28\left(\mathrm{~m}, H_{\mathrm{e}}(\mathrm{II})\right), 5.71$ (br s, $\left.H_{\mathrm{e}}(\mathrm{III})\right), 6.97-7.81(\mathrm{~m}$, $\left.H_{\text {arom }}\right)$ ppm. Ratio I:II:III $=100: 66: 37 .{ }^{13} \mathrm{C} \mathrm{NMR}\left(100 \mathrm{MHz}, \mathrm{CDCl}_{3}\right): 31.38,31.44,34.14,34.77$, 35.21 , 37.41, 43.90, 47.35, 47.64, 47.83, 53.48, 109.75, 109.88, 109.97, 119.79, 120.14, 120.27, $120.46,122.21,122.74,122.96,123.39,123.49,123.90,124.30,126.36,126.40,126.58,127.05$, $127.25,128.17,128.59,128.68,128.98,128.99,129.09,129.31,130.62,130.92,131.18,132.50$, $135.89,135,97,149.04,167.59,168.06,172.10,175.10,175.60,175.81 \mathrm{ppm}$. MS (EI, $70 \mathrm{eV})$ : $\mathrm{m} / \mathrm{z}(\%)=566$ (20.37) $[\mathrm{M}]^{+}, 418$ (16.85), 392 (100.00), 271 (16.05), 246 (19.89), 93 (7.70). Anal. Calcd. for $\mathrm{C}_{35} \mathrm{H}_{26} \mathrm{~N}_{4} \mathrm{O}_{4}$ : C 74.19, $\mathrm{H}$ 4.63, N 9.89. Found C 74.32, H 4.90, N 10.11.

* The resolution was worse than in the case of $\mathbf{6 a}$, and only two (instead of four) signals were observed.

\section{References and Notes}

1. (a) Herd, K. J. In Houben-Weyl, 4th Edn., Vol. E6b1; Kreher, R. P., Ed.; Thieme: Stuttgart, 1994, pp 451-545. (b) Bonnett, R.; North, S. A. Adv. Heterocycl. Chem. 1981, 29, 341. (c) Bonnett, R.; Brown, R. F. C.; Smith, R. G. J. Chem. Soc., Perkin Trans. I 1973, 1432. (d) Kovtunenko, V. A.; Voitenko Z. V. Russ. Chem. Rev. 1994, 63, 997. (e) Babichev, F. S.; Kovtunenko, V. A. Khimija isoindola (Chemistry of isoindole); Naukova Dumk: Kiev, 1983; Chem. Abstr. 1984, 101, 23311.

2. (a) Kovtunenko, V. A.; Voitenko, Z. V.; Tyltin, A. K.; Turov, A. V.; Babichev, F. S. Ukr. Khim. Zh., 1983, 49, 1287; Chem. Abstr. 1984, 100, 173943. (b) Kovtunenko, V. A.; Voitenko, Z. V.; Koutcherenko, T. T.; Turov, A. V.; Tyltin, A. K.; Babichev, F. S.; Khim. 
Geterotsikl. Soedin, 1990, 190; Chem. Abstr. 1990, 113, 39657. (c) Kovtunenko, V. A.; Kucherenko, T. T.; Voitenko, Z. V.; Tyltin, A. K.; Babichev, F. S. Ukr. Khim. Zh. 1988, 54, 186; Chem. Abstr. 1988, 109, 110287. (d) Kovtunenko, V. A.; Kucherenko, T. T.; Voitenko, Z. V.; Ukr. Khim. Zh. (Russian Ed.) 1992, 58, 1035; Chem. Abstr. 1993, 119, 27957. (e) Kovtunenko, V. A.; Kucherenko, T. T.; Voitenko, Z. V.; Tyltin, A. K.; Babichev, F. S. Ukr. Khim. Zh. (Russian Ed.) 1989, 55, 52; Chem. Abstr. 1984, 101, 23311. (f) Veber, D. F.; Lwowski, W. J. Am. Chem. Soc., 1964, 86, 4152-4158. (g) Kreher, R.; Use, G. Heterocycles, 1982, 19, 637. (h) Simons, S.; Ammon, H.; Doherty, R.; Johnson, F. J. Org. Chem., 1981, 46, 4739.

3. (a) Voitenko, Z. V.; Egorova, T. V.; Kovtunenko, V. A. Chem. Heterocycl. Comp. 2002, 38, 1019. (b) Voitenko, Z. V.; Pocholenko, A. A.; Chkarov, O. O.; Kovtounenko, V. A.; Babichev, F. S. Chem. Heterocycl. Comp. 2002, 38, 190. (c) Voitenko, Z. V.; Pocholenko, O. A.; Chkarov, O. O.; Shishkin, O. V.; Shishkina, S. V.; Dall'Ava, A.; Vedrenne, M.; Sanchez, M.; Wolf, J.-G. Eur. J. Org. Chem. 2001, 1401. (d) Voitenko, Z. V.; Samoilenko, V. P.; Kovtounenko, V. A.; Gurkevich, V. Yu.; Tyltin, A. K.; Shcherbakov, M. V.; Shishkin, O. V. Chem. Heterocycl. Comp. 1999, 35, 600. (e) Samoylenko, V. P.; Voitenko, Z. V.; Donnadieu, B.; Bonnet J.-J.; Tetrahedron 2002, 58, 6103. (f) Voitenko, Z. V.; Samoylenko, V. P. Chem. Heterocycl. Comp. 2002, 38, 197. (g) Voitenko Z.V. New rearrangements in cycloaddition of annelated at $a$ side isoindoles: stereochemical phenomena and intermediary products. Thesis. Kyiv, 2006.

4. (a) Kovtunenko, V. A.; Voitenko, Z. V.; Sheptun, V. L.; Tyltin, A. K.; Chernega, A. I.; Struchkov, Yu. T.; Babichev, F. S. Khim. Geterotsikl. Soedin. 1984, 1497; Chem. Abstr. 1985, 102, 45191. (b) Kovtunenko, V. A.; Voitenko, Z. V.; Sheptun, V. L.; Savranskii, L. I.; Tyltin, A. K.; Babichev, F. S. Ukr. Khim. Zh. (Russian Ed.) 1985, 51, 976; Chem. Abstr. 1986, 104, 167711. (c) Kovtunenko, V. A.; Voitenko, Z. V.; Sheptun, V. L.; Savranskii, L. I.; Tyltin, A. K.; Babichev, F. S.; Khim. Geterotsikl. Soedin., 1987, 5, 646; Chem. Abstr. 1988, 108, 111421.

5. Lyaskovskyy, V. V.; Voitenko, Z. V.; Kovtunenko, V. A. Chem. Heterocycl. Comp., in press.

6. Burgi, H.B.; Dunitz, J.D. "Structure Correlation", vol. 2, VCH: Weinheim, 1994.

7. Voitenko, Z. V.; Pokholenko, O. A.; Kovtunenko, V. O.; Karbovs'ka, R. B.; Tyltin, A. K. Visnik Kiiv. Un-tu, Ser. Khim. 2003, 40, 90.

8. Irick, G., Jr. J. Heterocycl. Chem., 1970, 7, 33. 